\title{
SATIP6 : NEXT GENERATION SATELLITE SYSTEM DEMONSTRATOR
}

\author{
Olivier Alphand ${ }^{1}$, Pascal Berthou ${ }^{1}$, Thierry Gayraud ${ }^{1}$, Sébastien Josset $^{2}$, \\ Eddy Fromentin ${ }^{3}$ \\ ${ }^{1}$ LAAS-CNRS, Toulouse, France ; ${ }^{2}$ Alcatel Space, Toulouse, France ; ${ }^{3} A Q L$, Rennes, France
}

\begin{abstract}
Owing to its modular design and implementation, the SATIP6 satellite emulation platform is able to emulate a transparent DVB-RCS (Digital Video Broadcasting - Return Channel via Satellite) system dimensioned around a single Hub, or a system using a regenerative satellite with an on-board switching matrix only by changing some configuration files. After a presentation of the SATIP6 project and architecture, we detail emulation techniques and the QoS architecture that was finally adopted.
\end{abstract}

Key words: IPv6, DVB-RCS, DAMA, Emulation, QoS

\section{INTRODUCTION}

Evaluating performances over real data links or network is often costly, even impossible for systems in development phase. Simulation and emulation both provide the opportunity to evaluate performances, at low cost, on more or less realistic systems. When simulation needs a complete modelisation of the systems from applications to physical network and operates in virtual time, emulation is more demonstrative since real applications can be deployed over the model describing transfer characteristics, delay and error behavior for instance.

For these reasons, the choice was made for the IST SATIP $6{ }^{1}$ project to set up a satellite emulation platform to demonstrate the network and application services integration on next generation satellite systems and the possibility to interoperate with terrestrian networks.

This paper first gives a concise overview of the SATIP6 project, then briefly sums up the main functionnalities of DVB-RCS ${ }^{4}$ satellite systems and finally describes the experimental platform configuration that was adopted. 
The second section depicts the design and the implementation of this full DVB-S/RCS emulated satellite platform. It mainly focuses on the satellite Physical and Data Link layers emulation techniques. The final section deals with the QoS Architecture that was retained to provide differentiated QoS on a satellite network

\section{THE SATIP6 PROJECT}

\subsection{SATIP6 objectives}

The aims of SATIP6 projects were to evaluate and demonstrate key issues of the integration of satellite-based access networks into the Internet in order to offer multimedia services over wide areas. IP has served as the common denominator to allow interoperability for services and transport technology within integrated networks.

Most immediate technical issues facing satellite broadband access in the coming years have been examined, namely the functions to be implemented in the protocol layer between physical medium access and the IP Layer (i.e. typically layer 2). The project has considered two stages:

- short-to-medium term focussing on better adaptation of DVB-RCS access for IP services with current satellites

- longer term in which protocols more optimised for IPv6 will be introduced, with next generation satellites (including both transparent and regenerative payloads)

This paper is related to the second step. In this step, the shorter term solutions defined for IP/DVB-RCS have been extended and adapted for more advanced satellite systems supplemented with mesh connectivity (directly between users) via transparent or regenerative payloads (with onboard packet switching). In addition, satellite integration into NGN (Next Generation Networks), based on IPv6 and associated with a range of advanced features has been targeted : Security ${ }^{5}$, Mobility, Performance Enhancing Proxy, Multicast. Moreover Layer 2 protocols, such as "IPdedicated" has been defined for more optimized transport of IPv6 over satellite compared with MPLS).

One of the starting points of the SATIP6 project was the architecture developed in the IST project BRAHMS ${ }^{7}$, and adopted within the ETSI BSM group. The SATIP6 scenario, shown in Fig. 1, consists in a geostationary satellite network with onboard switching capabilities, Ka MF-TDMA (Multiple Frequency- Time Division Multiple Access) uplinks and Ku TDM (Time Division Multiplexed ) downlinks. Satellite Terminals (STs) provide single PC or LANs with the access to the network, while Gateways (GTs) 
allows the connection with Internet core networks. The uplink access from each ST is managed through DVB-RCS (Return Channel via Satellite) interface, while transmission from GTs is implemented through DVB-S interfaces. STs and Gateways are boundary devices between the satellite and terrestrial links and play an important role in access to satellite resources and hence in QoS provision. In the SATIP6 architecture both devices implement IP routing and has an IP interface on the satellite segment.

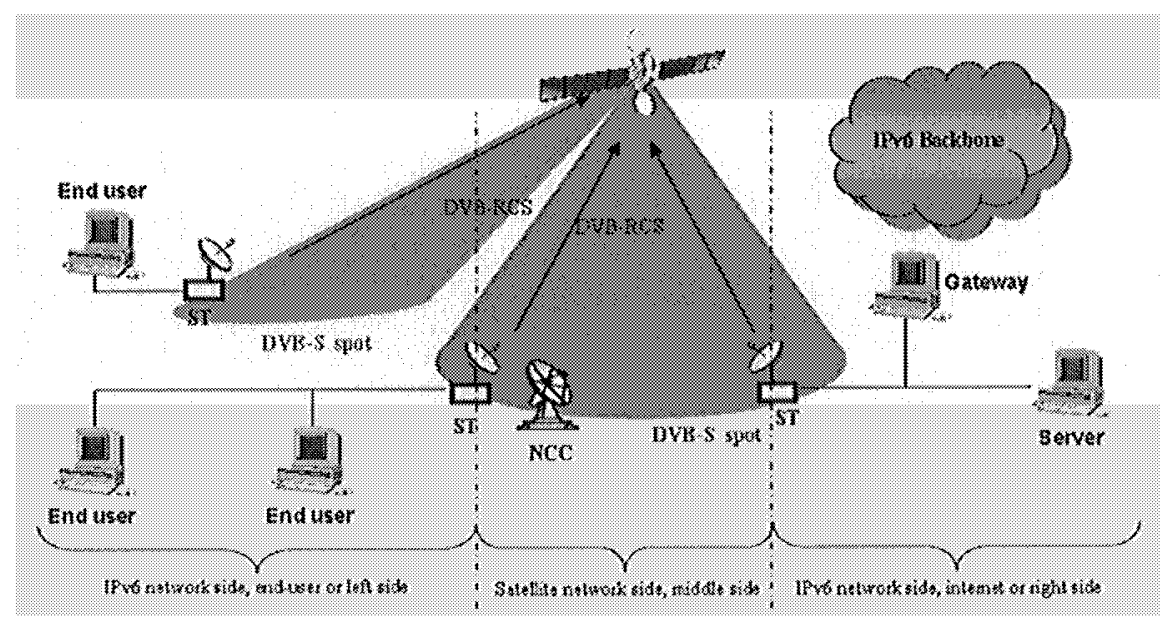

Figure 1. SATIP6Architecture

Three main components have to be distinguished in the satellite network side (middle): the Satellite Emulator (SE), the Satellite Terminals (ST) and the Network Control Center (NCC)

The SE is a regenerative satellite that use IP-dedicated ${ }^{6}$ as an optimization layer for switching frames. Functionally ST acts as routers. That is to say that Satellite Network is considered as a special link in classical network point of view. By using special mechanisms (IP-dedicated address resolution) they are able to transmit IP packets from one side of the IP network to the other side of the IP network.

\subsection{Experimental platform}

To validate our proposal, a testbed has been designed. The testbed that has been developped is able to emulate the three main parts of a satellite communication system (SE, ST, NCC). It is described in Fig. 2. The middle side interconnects two user LANs (individual user, company subsidiaries ...) and the right LAN (LAN 3), acting as an Internet Service Provider site or as the company headquarters. LAN 3 provides also Internet interconnection, 
using native IPv6. Each LAN consists of one Satellite Terminal (ST) and several user workstations (WS).

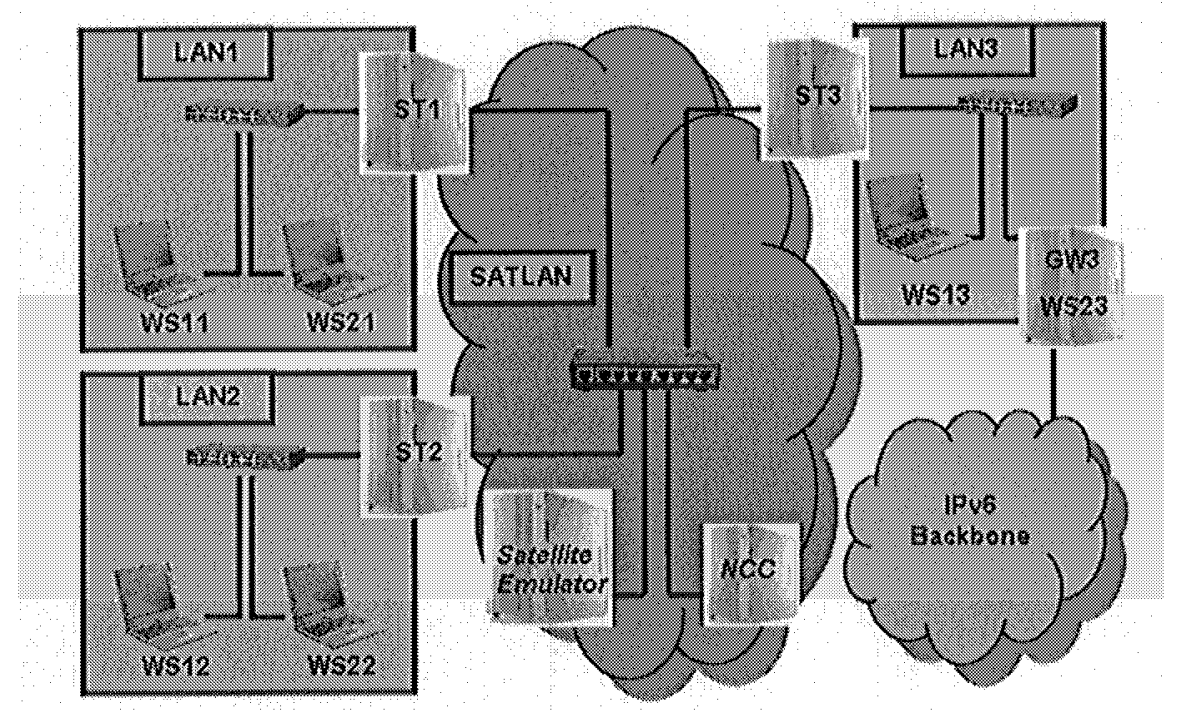

Figure 2. Full SATIP6 testbed

\section{THE SATIP6 ARCHITECTURE IMPLEMENTATION}

\subsection{The Satellite System Emulation Tools}

In order to have the most modular platform and so preserves room for future evolution, stringent requirements were fixed before the development phase.

At first, the aim was to take advantage of a linux system (Red Hat 9 Distribution) which natively supports Ipv6 and a wide panel of IPv6 applications (Apache as HTTP Server, Mozilla as HTTP Client, $V_{s f t p d}$ as FTP Server, Gnomemeeting for Videoconferencing, VideoLanClient for Videostreaming).

Then the next step was to decide how to implement the Satellite Protocol stack and the main satellite systems functionnalities. We choose to directly reuse a derived GPL product for the runtime. It is called Margouilla ${ }^{2}$, a C++ runtime that provides platform independent messaging, a set of common blocs ready to use (IP/ATM/Ethernet layers...), a graphical editor with SDL design style and utilities package offers basic tools, such as Configuration 
file and logging mechanisms for error and debug messages. For the purpose of SATIP6, Margouilla at first enables the running of automatically generated piece of codes compiled from an SDL description. Secondly, its runtime provides us with a multi-platform synchronisation toolkit and finally stands for a formal platform for collaborative development.

\subsection{The emulated functionnalities}

The bloc that were developped within the Margouilla runtime are :

- The satellite carrier package is responsible for the different satellite carriers emulation on top of Ethernet (DVB-RCS, DVB-S and Signaling Channels) and the simulation of typical satellite bit errors and delay.

- The $D V B-S / R C S$ package implements a framing structure compliant with the DVB-S/RCS standards. and fills DVB-RCS frames with ATM-like cells coming from the IP-dedicated layer. In order to achieve proper QoS, this layer manages synchronisation and queues according to the authorisations a DAMA algorithm delivers.

- The DAMA package implements the DAMA algorithms used to manage the satellite ressources allocation at layer 2 .

- The IP Dedicated package implements the IP dedicated layer which is responsible for segmentation and reassembly functionnalities and for a specific tagging mechanism targeted towards IP. It also implements a dynamic address resolution protocol in order to use that mechanism.

- The IP QoS Package implements common mechanisms to enable differentiation at this level. It treats packets incoming from IP network and forwarded on the DVB-DCS uplink according to a committed QoS behaviour and is in charge of discriminating, regulating and scheduling this traffic in to 3 classes of Service (Real-Time, non Real-Time and Best Effort).

A detailed description of the way some layers are emulated is done through the following paragraphs.

\subsubsection{The Satellite Carrier Emulation}

The satellite carrier emulation is designed to operate on top of Ethernet frames and for each satellite channel corresponds an Ethernet multicast address. Ethernet was chosen for its native broadcast abilities and also for its high bandwidth capacities.

For each spot, we distinguish at least 4 channels :

- There is a dedicated channel for each data DVB-S flow spot descending from the satellite (one channel per DVB-S flow per spot) 
- There is a dedicated channel for each data DVB-RCS flow on each spot ascending to the satellite

- There are two dedicated channels for control frames (connection request, connection confirm, TBTP, ...) one for the ascending flow and one for the descending flow

\subsubsection{The Satellite Link Emulation}

The main functionnality of the SE is its satellite link emulation module which simulates in real time the moduling/coding part thanks to precalculated BER files. The error model is based on precalculated data files from IST BRAHMS project. In addition to inject bit errors, the satellite emulator introduces delay and jitter that can be also tuned. The default values were put to $250 \mathrm{~ms}$ with no jitter.

\subsubsection{The DVB-RCS transmission emulation}

This final section deals with the QoS Architecture and especially the resource allocation procedure that was adopted in the SATIP6 demonstrator. Before detailing this procedure, we must precise two techniques on which the allocation procedure is based : the synchronisation emulation, the DVBRCS frame transmission emulation over Ethernet.

The synchronisation emulation is carried out by emitting Ethernet frame at fixed instant on all ST (super frame tick) and then internally by awaking processes each frame ticks $(50 \mathrm{~ms})$ to send already queued packet. In this process, we are limited by a $10 \mathrm{~ms}$ granularity imposed by Linux. The default reference values on which the allocation procedure is based are :

- The superframe composed of 10 internal frames

- Frame duration of $50 \mathrm{~ms}$

- Periodicity of ATM cells allocation on a super frame basis: i.e. each $500 \mathrm{~ms}$.

The reproduction of a typical DVB-RCS allocation structure based on ATM bursts cannot be exploited using classical ethernet hardware interface since there is no possibility for a given station to fill an ethernet frame emitted by another station. Therefore, we have to use Ethernet frames to transport our DVB frames. However, transmitting an ethernet frame every time a RCST should fill its dedicated timeslots of the current superframe generates too much overhead. On the opposite, transmitting the whole pool of ATM cells allocated by the NCC for this superframe in a unique Ethernet frame leads to a burst effect on Ethernet layer that causes loss of packets. Thus ATM cells allocated to a RCST for a superframe are shared among DVB-RCS frames encapsulated in Ethernet frames that are efficiently 
distributed over the superframe duration. The efficiency is directly linked to the number of ATM cells to transmit. For instance, very small isolated traffic (TCP Acknowledgment or ping) are not spanned on the length of the superframe but transmitted as soon as possible.

Here was detailed the ATM cell pool repartition into a ST DVB-RCS frame during a superframe duration. However a question still remains : which mechanisms is used to provide an efficient satellite DVB-RCS resource allocation? This is the point of the last section which provides a description of the SATIP6 QoS Architecture.

\subsection{The QoS Architecture}

In order to illustrate it, the figure 3 gives a detailed view of the architecture of IP and MAC Layers of one ST DVB-RCS part.

\subsubsection{IP Layer}

The IP Layer component defining QoS classes and implementing buffer and bandwidth management mechanisms to assure fair allocation of bandwidth among contending users/applications and to control queuing delays.

Differentiated Services are performed through 3 Classes of Services (RT, NRT and BE):

- Real Time (VoIP, Videoconference) : delay and jitter sensitive traffic with hard constraints that must take benefit from the guarantees provided by a static capacity assignment

- Non Real Time (Multiplayer gaming, Videostreaming): tolerant to delay, jitter and loss threshold. A minimum bandwidth is needed and therefore can resort to use dynamic capacity assignment.

- Best Effort (Web, E-Mail ...): All the traffic that can recover from medium losses and support high delay

RT and NRT traffic is policed and shaped while BE is not policed. 


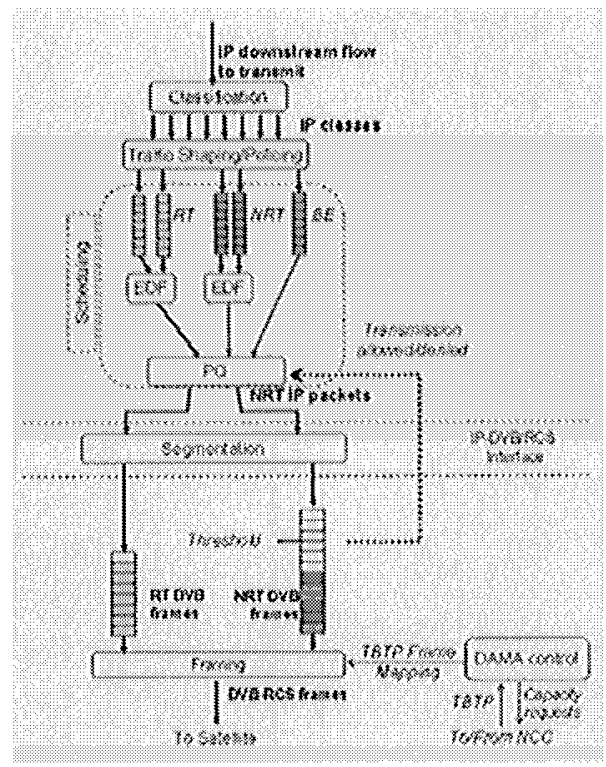

Figure 3. SATIP6 QoS Architecture

\subsubsection{MAC Layer}

The main role of the MAC Layer DAMA component is to manage the contented access to uplink bandwidth.

Thus, the MAC layer defines two classes of service $(\mathrm{CoS})$ through the Real-Time and the non Real-Time DVB-RCS queues. They are based on a different allocation schemes. On one side, the DVB-RCS RT frame emission only consumes statically assigned slots when, on the other side, the number of NRT frames transmitted during a superframe is only linked to the dynamic allocated slots thanks to the DAMA.

Finally, in order to prevent MAC-layer buffer overflows, the scheduler no more forward IP Packets to the MAC Layer if the NRT MAC queue length exceeds a predefined threshold.

The allocation procedure is at last described and give some hints about the resource management optimization process that was used in the SATIP6 project.

\subsubsection{The Allocation Procedure}

The allocation procedure implementation in the demonstrator is described below: 
- At log-on STs request a fixed RT bandwidth, which is allocated by subtracting the asked capacity to the total bandwidth available. There is no further allocation demand for that kind of traffic.

- STs mainly do their request according to the number of ATM cells that feeds the NRT MAC queue during the previous superframe and the NRT MAC buffer queue length at the beginning of the current superframe ${ }^{3}$. In particular there is an overprovision factor that takes into account sizes of queues during the 2 last super frames. Its goal is to enhance capacity requests according to the traffic recently experienced, it helps voiding the queues if congestion occurred. It is governed by a factor $(1-\alpha)$. If $\alpha$ is equal to 0 there is maximal overprovision. If it is equal to 1 there is no overprovision.

- Each CR is launched at the beginning of a super frame. Each CR is then delayed by the satellite emulator and reach NCC $250 \mathrm{~ms} \pm 10 \mathrm{~ms}$ (due to $10 \mathrm{~ms}$ awoke rate in the Satellite Emulator as explained above) after. CRs are processed by NCC upon arrival.

- NCC computes allocations at the beginning of each super frame according to an internal SACT table based on $\mathrm{CR}$ received. Authorisations are sent back to STs using a TBTP table (if $f c a>0$, even not requesting logged on station will receive extra-bandwidth if available).

- Upon reception of a TBTP, allocation are stored by ST and used in the next super frame

\section{CONCLUSION}

This article has presented the emulation platform developped during the SATIP6 project. It aims at emulating the most accurately an IP over DVBRCS satellite network. Both IPv4 and IPv6 are implemented. The difference between an emulator and a simulator is the possibility to use real systems and real applications on top of the emulator. Thanks to this platform, evaluation of the quality of service experienced by interactive multimedia applications when using a new generation satellite network was possible. It definitly refutes the bias that interactive multimedia applications do not tolerate with the long delay of satellite networks. However these networks need a fine resource management and an efficient quality of service signalisation

Thus the first evaluations conducted on our platform has been proved to be complex and lot of works is still needed to find all the ins and outs of the QoS over new generation satellite networks. 
Besides the platform involves for the moment a lot of computers and is hard to be configured. We are working now to reduce the number of computer from 5 to 1 , thanks to the modular architecture provided by Margouilla. This will be an efficient tool for satellite networks research.

\section{REFERENCES}

1. IST SATIP6 Project (Contract IST-2001-34344), Home Page on http://satip6.tilab.com

2. Margouilla C++ Runtime : http://casoftware,free.fr/margouilla

3. E.Impenba, T.Inzerilli, Epaone, A.Pietrabissa, G.Tarquini. "QoS Support for Interactive Communication with DVB/RCS Satellites", Universta di Roma "La Sapienza", ISCC'04

4. EN 301 790: "Digital Video Broadcasting (DVB) ; Interaction channel for satellite distribution systems"

5. L. Duquerroy, S. Josset, O.Alphand, P.Berthou, T. Gayraud "SatIPSec : an optimized solution for securing multicast and unicast satellite transmissions", AIAA'04

6. S.Combes, S.Josset, P.Very, I.Buret, T.Zein, "Network Scenario for IP-Dedicated Satellite Access Scheme", 8th Ka-Band Utilization Conference, 25-27 September 2002 Baveno (Italy)

7. Brahms IST project (Contract IST-99-10440), http://brahms.tilab.com 